RASĀYAN J. Chem.

Vol. 13 | No. 1 |529 - 534| January - March | 2020 ISSN: 0974-1496 | e-ISSN: 0976-0083 | CODEN: RJCABP

\title{
FORMULA COMPARISON OF NANOEMULSION AND CREAM CONTAINING MICONAZOLE NITRATE: PENETRATION TEST USING FRANZ DIFFUSION CELLS AND ANTIFUNGAL ACTIVITY TEST ON Tricophyton mentagrophytes, Microsporum canis AND Candida albicans
}

\author{
H. L. Maha ${ }^{1, *}$ and Masfria ${ }^{2}$ \\ ${ }^{1}$ Department of Pharmaceutical Technology, Faculty of Pharmacy, Universitas Sumatera Utara, \\ Padang Bulan, Medan, 20155, Indonesia \\ ${ }^{2}$ Department of Chemistry Pharmacy, Faculty of Pharmacy, Universitas Sumatera Utara, Padang \\ Bulan, Medan, 20155, Indonesia \\ *E-mail: hetty_maha03@usu.ac.id
}

\begin{abstract}
Miconazole nitrate is one of the antifungal agents applied to the skin. Miconazole nitrate has a low bioavailability if it implements for oral treatment due to poor solubility and has low absorption, therefore miconazole nitrate implemented as the antifungal agent for topically. In addition, the main problem of a drug is the treatment of topical for poor skin penetration ability. The aim of the current study was the comparison of the penetration and the antifungal activity of both dosages. Nanoemulsion and cream containing miconazole nitrate were made in three formulas namely $1 \%, 1.5 \%, 2 \%$ and one formula as a blank (without miconazole nitrate). The nanoemulsion is formulated with a low energy emulsification method. The formula was then evaluated for its particle size, drug release profile and antifungal activity test against Tricophyton mentagrophytes, Microsporum canis and Candida albicans. The particle size of nanoemulsion is $102.36 \mathrm{~nm}, 112.23 \mathrm{~nm}$ and $117.52 \mathrm{~nm}$ sequentially. The in-vitro release profile of nanoemulsion is between $65.81 \%$ to $71.09 \%$ while the cream is between $35.94 \%$ to $41.84 \%$ for 12 hours. Nanoemulsion contains miconazole nitrate $2 \%$ has an effective effect for the Cream $2 \%$ that number includes inhibit zone $28.2 \pm 1.15 \mathrm{~mm}$ for Tricophyton mentagrophytes, $21.0 \pm 0.77 \mathrm{~mm}$ for Microsporum canis and $23.7 \pm 0.90$ $\mathrm{mm}$ for Candida albicans. From the current study, it can be concluded that nanoemulsion dosage forms result in penetration and antifungal activity is better than cream dosage forms. Preparations nanoemulsion $2 \%$ resulted in improving the most penetration and antifungal activity.
\end{abstract}

Keywords: Nanoemulsion, Cream, Miconazole Nitrate, Franz Diffusion Cell, Antifungal.

(C) RASĀYAN. All rights reserved

\section{INTRODUCTION}

Topical treatment of fungal infections has various advantages, namely target to the infection area, reduce the risk for systemic side-effects, increase drug effectiveness and patient compliance. ${ }^{1}$ The efficiency of topical antifungal treatment is depending on the penetration of the drug by the target tissue. Therefore, the effective concentration of the drug must be delivered to the site of infection. ${ }^{2}$ Miconazole nitrate (Fig.-1) is a broad-spectrum antifungal agent of the imidazole group. ${ }^{3}$ These antifungals are fungicides that implemented for a treatment of topical and transdermal fungal infections. ${ }^{4}$ The drug is primarily implemented as a topical treatment for cutaneous mycoses. The bioavailability of miconazole nitrate is very low when implemented orally. But a presents problem for the treatment of cutaneous diseases due to the topical application is poor skin penetration capability. ${ }^{3}$ Various new methods like complexation method with cyclodextrin, submicron emulsion, and chewing gum for buccal administration has also been implemented in the development of the miconazole nitrate formulation but the drug content in the blood remains low. ${ }^{5}$

Rasayan J. Chem., 13(1), 529-534(2020)

http://dx.doi.org/10.31788/RJC.2020.1315553

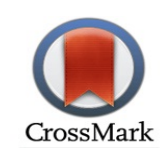


RASĀYAN J. Chem.

Vol. 13 | No. 1 |529 - 534| January - March | 2020

To design effective miconazole nitrate formulations have known to become the main challenge due to efficacy able to severely limit by instability or poor solubility of a vehicle. ${ }^{6}$ A specific pharmaceutical preparation that can be implemented in drug delivery systems to resolve the problems is the nanoemulsion drug delivery system, which is applied to increase the solubility and bioavailability for lipophilic drugs. ${ }^{6}$ Nanoemulsions are colloidal dispersion systems that arise to the stability of thermodynamical conditions, collected from two immiscible liquids mix together by emulsifying agents (surfactants and co-surfactants) to form the single phase. ${ }^{7}$ Their long-term stability, easy preparation, and higher solubilization of drug molecules made it promising to become the drug delivery system. ${ }^{8}$ Nanoemulsion also has the potential as a carrier in topical treatment because it is able to optimize the dispersion of active substances in the skin layer. ${ }^{4}$ Nanoemulsion does not have creaming, sedimentation, and flocculation or coalescence compare to the macroemulsion. ${ }^{21}$

\section{Materials}

\section{EXPERIMENTAL}

The materials in the current study are miconazole nitrate (from Kimia Farma Watudakon Jombang East Java Indonesia), oleic acid, tween 80, PEG 400, cetyl alcohol, propylene glycol, vaseline, glyceryl monostearate, TEA, sodium metabisulfite, aqua dest, potato dextrose agar (PDA), Mc. Farland Solution, Physiological $\mathrm{NaCl} 0.9 \%$. All the ingredients were of analytical grade.

\section{Formulation of Miconazole Nitrate Nanoemulsion}

Miconazole nitrate nanoemulsion was prepared by low energy emulsification method. Oil-in-water nanoemulsions were formulated at varied concentrations of miconazole nitrate. Nanoemulsion formulation in the absence of a miconazole nitrate was also prepared. The emulgators implemented were Tween 80 and PEG 400. The water phase which includes sodium metabisulfite and aquabidestilata was mixed and stirred with the help of magnetic stirrer until homogeneous. The oil phase was added to the water phase while adding the emulgator mixture and stirring using a magnetic stirrer, then the mixture was homogenized by a homogenizer that the speed was set at $1000 \mathrm{rpm}$ for 60 minutes after that sonicated for 30 minutes until clear nanoemulsions were formed. ${ }^{9}$ The formula design of miconazole nitrate nanoemulsion is shown in Table-1.

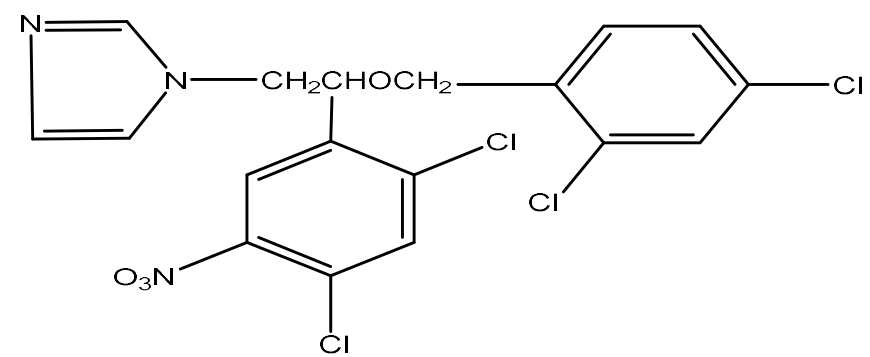

Fig.-1: Structure of Miconazole Nitrate

Table-1: Compositions of Miconazole Nitrate Nanoemulsion

\begin{tabular}{c|c|c|c|c}
\hline \multirow{2}{*}{ Composition } & \multicolumn{3}{|c}{ Formula } \\
\cline { 2 - 5 } & F1A & F2A & F3A & F4A \\
\hline Miconazole nitrate (\% v/v) & - & 1 & 1.5 & 8 \\
\hline Oleic acid (\% v/v) & 8 & 8 & 8 & 25 \\
\hline Tween 80 (\% v/v) & 25 & 25 & 5 & 5 \\
\hline PEG 400 (\% v/v) & 5 & 5 & 0.1 & 0.1 \\
\hline Sodium metabisulfit (\% v/v) & 0.1 & 0.1 & 100 & 100 \\
\hline Aquabidest ad (ml) & 100 & 100 & & \\
\hline
\end{tabular}

\section{Note:}

F1A: Nanoemulsion without containing Miconazole Nitrate (Blank)

F2A: Nanoemulsion containing $1 \%$ of Miconazole Nitrate

F3A: Nanoemulsion containing $1.5 \%$ of Miconazole Nitrate

F4A: Nanoemulsion containing $2 \%$ of Miconazole Nitrate 


\section{Particle Size Measurement of Nanoemulsion}

Droplet sizes of nanoemulsion were measured by dynamic light scattering (DLS) by non-invasive back scatter technology. An experiment was conducted at $25^{\circ} \mathrm{C}$ on Zetasizer Nano ZS (Malvern Instruments, UK) provided by the helium-neon laser operating at $633 \mathrm{~nm}$. The DLS result was analyzed by a method of Cumulants in which the intensity correlation function was related to a diffusion coefficient and eventually converted to a hydrodynamic size. The width of size distribution was referred to as a polydispersity index (PDI). The data obtained was represented as an average of three determinations. ${ }^{10}$

\section{Cycling Test}

The nanoemulsion preparation was stored at $4^{\circ} \mathrm{C} \pm 2^{\circ} \mathrm{C}$ for $24 \mathrm{~h}$, then into the oven at $40^{\circ} \mathrm{C} \pm 2^{\circ} \mathrm{C}$ for the next $24 \mathrm{~h}$. This treatment is one cycle. The experiment was repeated for 6 cycles. After a cycling test, see the physical condition for nanoemulsion before then after the test. ${ }^{11}$

\section{Centrifugation Test}

The sample was inserted into a centrifugation tube then inserted into a centrifugator with a spin speed $3800 \mathrm{rpm}$ for $5 \mathrm{~h}$. Treatment results are equivalent to a gravity effect for 1 year. After centrifugation was observed, the physical condition of the preparation compared before and after the test. ${ }^{12}$

\section{Preparation of Miconazole Nitrate Cream}

All necessary materials were weighed. The materials were separated into two groups: The oil and water phases. The oil phase consists of vaseline, stearic acid, and cetyl alcohol melted over a water bath with a temperature of $70-75^{\circ} \mathrm{C}$. After a perfect melt was implemented, miconazole nitrate into it. In a separate container, aqueous phases comprising aqua dest, propylene glycol, and TEA are dissolved in hot water. On a continuous phase, the water in a hot melt then slowly added to the oil phase with constant stirring at the temperature more or less $70^{\circ} \mathrm{C}$, until a cream mass was obtained. ${ }^{11}$ The formula design of miconazole nitrate cream is shown in Table-2.

Table-2: Compositions of Miconazole Nitrate Cream

\begin{tabular}{c|c|c|c|c}
\hline \multirow{2}{*}{ Composition } & \multicolumn{4}{c}{ Formula } \\
\cline { 2 - 5 } & F1B & F2B & F3B & F4B \\
\hline Miconazole nitrate (\% w/w) & - & 1 & 1.5 & 2 \\
\hline Vaseline (\% w/w) & 5 & 5 & 5 & 3 \\
\hline Cetyl alcohol (\% w/w) & 3 & 3 & 3 & 3 \\
\hline Stearic acid (\% w/w) & 3 & 3 & 3 & 3 \\
\hline Glyceryl monostearate (\% w/w) & 3 & 3 & 5 & 5 \\
\hline Propylene glycol (\% w/w) & 5 & 5 & 1 & 0.1 \\
\hline TEA (\% w/w) & 1 & 1 & 100 & 100 \\
\hline Sodium metabisulfite (\% w/w) & 0.1 & 0.1 & & 0.1 \\
\hline Aquabidest ad (g) & 100 & 100 & & \\
\hline
\end{tabular}

Note:

F1B: Cream without containing Miconazole Nitrate (Blank)

F2B: Cream containing $1 \%$ of Miconazole Nitrate

F3B: Cream containing $1.5 \%$ of Miconazole Nitrate

F4B: Cream containing $2 \%$ of Miconazole Nitrate

\section{In Vitro Skin Permeation Studies of Miconazole Nitrate Nanoemulsion and Cream}

The drug release kinetics studied implementing a modified Franz diffusion cell. Male rabbits weighing $1.8 \mathrm{~kg}$ were chosen for the in vitro studies. ${ }^{11}$ Hairs on the abdominal region were removed by the razor knife (Gillette Brand) with no break of the stratum corneum. ${ }^{23}$ During the experiment period, animals that anesthetized with chloroform and abdominal skin was established. The fatty material adhered to the dermis was carefully peeled off. Miconazole nitrate nanoemulsion was applied to the surface of the skin in the donor compartment, The samples were withdrawn at different time intervals as much as $0.5 \mathrm{~mL}$ from the receptor compartment and analyzing of drug content using a UV-Visible spectrophotometer. ${ }^{11}$ 
RASĀYAN J. Chem.

Vol. 13 | No. 1 |529 - 534| January - March | 2020

In Vitro Antifungal Activity Test of Miconazole Nitrate Nanoemulsion and Cream

Ditch plate technique implemented for the study. ${ }^{22}$ It is a technique applied for the evaluation of bacteriostatic or fungistatic activities of the compound. The nanoemulsion preparation as much as 0.05 gram were located on the ditch cut of a plate. Freshly prepared culture loops of Tricophyton mentagrophytes, Microsporum canis and Candida albicans were streaked across the agar at a right angle from the ditch to the edge of the plate. Triplo was repeated in the same way. ${ }^{13}$ When the incubation established for 5 to 7 days at $25^{\circ} \mathrm{C}$, the fungal growth was analyzed and then the diameter of the inhibit zone was measure horizontally and vertically. ${ }^{14}$

\section{Particle Size Measurement of Nanoemulsion}

\section{RESULTS AND DISCUSSION}

Droplet sizes of nanoemulsion were measured by dynamic light scattering (DLS) by non-invasive backscatter technology. Measurement results show on Table-3.

Table-3: Particle Size of Miconazole Nitrate Nanoemulsion

\begin{tabular}{c|c|c}
\hline Formula & Particle size & Intensity \\
\hline F2A & $102.36 \mathrm{~nm}$ & 0.51 \\
\hline F3A & $112.23 \mathrm{~nm}$ & 0.47 \\
\hline F4A & $117.52 \mathrm{~nm}$ & 0.71 \\
\hline
\end{tabular}

\section{Cycling Test}

All the formulas on the cycling test after 6 cycles, the nanoemulsion preparation remained weak yellow and odorless, and the cream preparations remained white and did not show phase separation.

\section{Centrifugation Test}

After all the formula was centrifuged at $3800 \mathrm{rpm}$ for 5 hours, both nanoemulsion and cream preparations did not show phase separation.

In Vitro Skin Permeation Studies of Miconazole Nitrate Nanoemulsion and Cream

The drug release kinetics studied using a modified Franz diffusion cell. This test is carried out to find out how much miconazole nitrate is able to penetrate by the skin barrier. The cumulative amount of penetrated miconazole nitrate shows in Fig.-2.

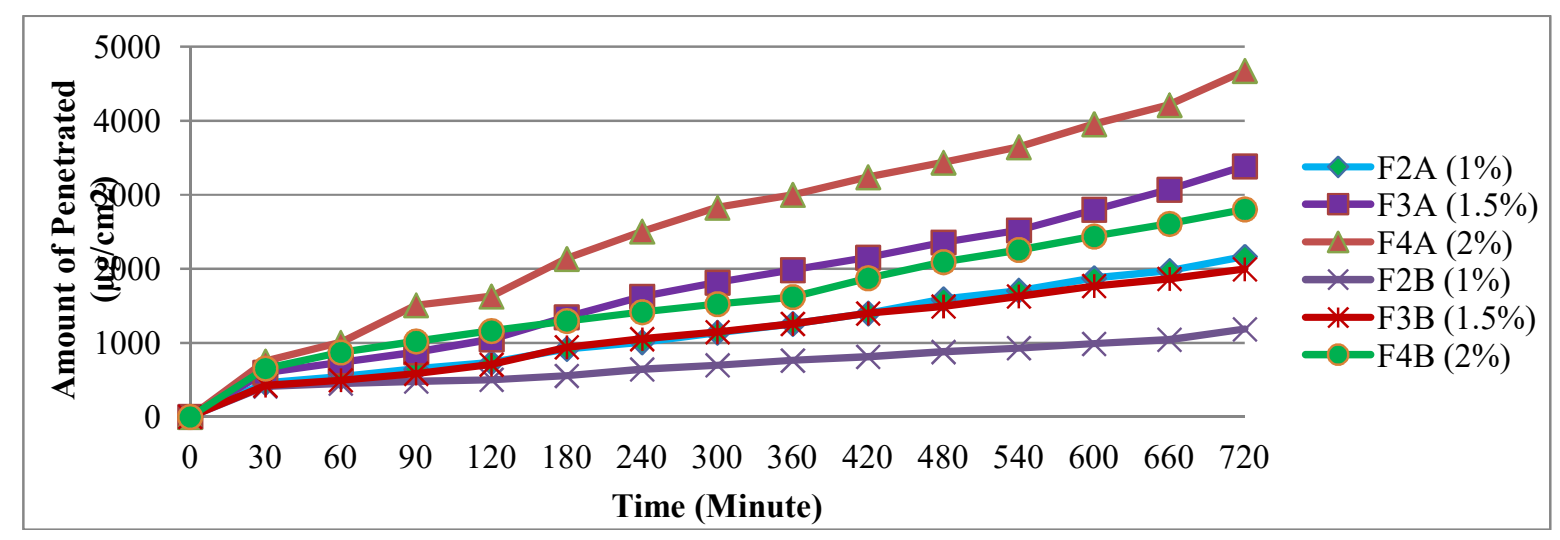

Fig.-2: The Cumulative Amount of Miconazole Nitrate Penetrated from the Nanoemulsion and Cream Preparations

Based on the Fig.-2 shows that the cumulative amount of miconazole nitrate penetrated by rabbit skin during the 720-minute penetration test of each nanoemulsion preparations in sequence are 2165.20 $\mu \mathrm{g} / \mathrm{cm}^{2}, 3387.81 \mu \mathrm{g} / \mathrm{cm}^{2}$ and $4677.38 \mu \mathrm{g} / \mathrm{cm}^{2}$ and from cream preparations are $1183.36 \mu \mathrm{g} / \mathrm{cm}^{2}, 1995.80$ $\mu \mathrm{g} / \mathrm{cm}^{2}$ dan $2805.24 \mu \mathrm{g} / \mathrm{cm}^{2}$. From these results, miconazole nitrate in nanoemulsion dosage form has a cumulative amount of penetration greater than the cream dosage form. This is because the nanoemulsion particle size is smaller than the cream which makes it easier for miconazole nitrate to penetrate. The small 
RASĀYAN J. Chem.

Vol. 13 | No. 1 |529 - 534| January - March | 2020

particle size of nanoemulsion increases the surface area of the emulsion system so that penetration is better. $^{15}$

\section{Comparison of Cumulative Percentage of Miconazole Nitrate Nanoemulsion and Cream Preparations}

The results obtained from the penetration of miconazole nitrate nanoemulsion preparations compared with cream preparations, as shown in Fig.-3.

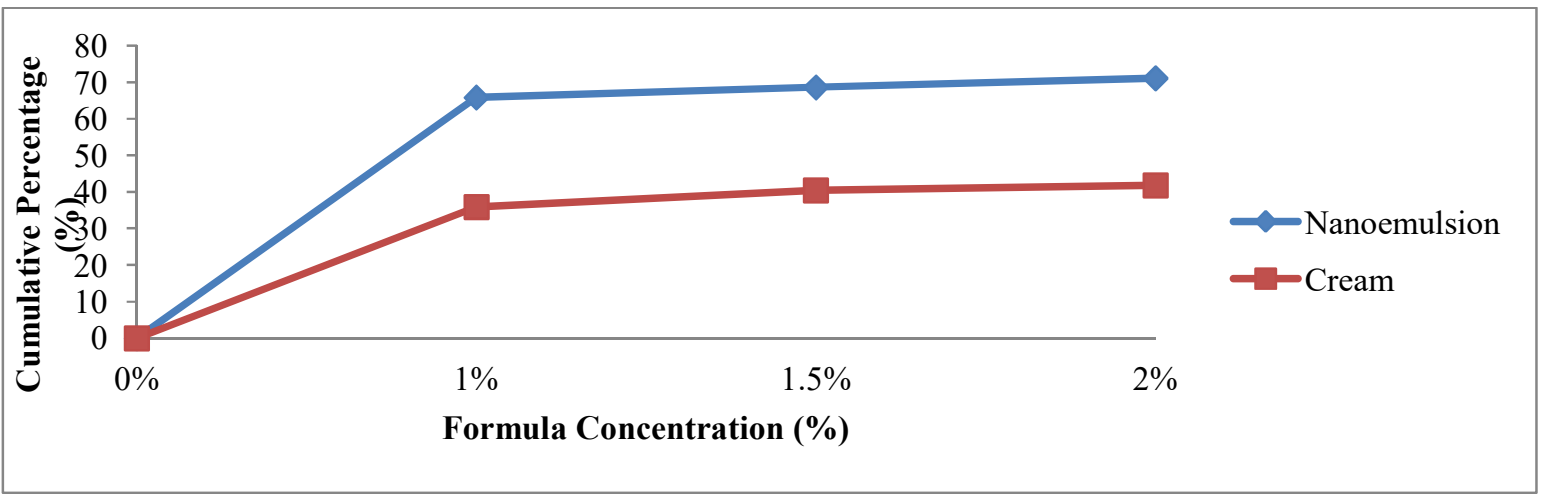

Fig.-3: Comparison of the Cumulative Percentage of Miconazole Nitrate Penetrated in the 720 Minute of Nanoemulsion and Cream Preparations.

Percentage of the cumulative amount of miconazole nitrate penetrated from each nanoemulsion preparation is $65.81 \%, 68.64 \%$ and $71.09 \%$ and from each cream formula are $35.94 \%, 40.44 \%$ and $41.84 \%$. Overall, it can be seen that the nanoemulsion formula produces a greater percentage of cumulative amounts compared to the cream formula, this is probably due to the nanoemulsion formula has a smaller particle size than the cream so that it can cross the rough skin surface and can increase drug penetration. ${ }^{16}$ Nanoemulsion preparations can help dissolve lipophilic drugs, thereby increasing drug penetration by the skin membrane. Nanoemulsion has also been reported to have higher skin permeation compared to other drug delivery systems such as microemulsions and liposomes. ${ }^{17}$

Besides that, it is also due to the use of tween 80 as a surfactant which is also a penetration increasing. ${ }^{18}$ The theory is proven by previous studies, comparing nanoemulsion spray with cream, in which the nanoemulsion penetration ability was greater because the smaller particle size obtained. ${ }^{19}$

\section{In vitro Antifungal Activity Test of Miconazole Nitrate Nanoemulsion and Cream}

The antifungal activity test of the formula observed by seeing the minimum inhibition using the punch hole method with a hole diameter of $6 \mathrm{~mm}$ on the agar media against Trycophyton mentagrophytes, Microsporum canis and Candida albicans. Table-4 showed the result of the antifungal activity test for nanoemulsion and cream miconazole nitrate. These results show that the antifungal activity of nanoemulsion is better than the cream dosage form because all concentrations are above the antimicrobial activity requirements with a range of $14-16 \mathrm{~mm} .{ }^{20}$

Table-4: Antifungal Activity Data of Formula

\begin{tabular}{l|c|c|c}
\hline \multirow{2}{*}{ Formula } & \multicolumn{3}{|c}{ Inhibitory Diameter (mm) } \\
\cline { 2 - 4 } & Trycophyton Mentagrophytes & Microsporum Canis & Candida Albicans \\
\hline Nanoemulsion (Blank) & $00.0 \pm 0.00$ & $00.0 \pm 0.00$ & $00.0 \pm 0.00$ \\
\hline Nanoemulsion 1\% & $20.4 \pm 1.10$ & $17.4 \pm 0.20$ & $17.1 \pm 1.37$ \\
\hline Nanoemulsion 1,5\% & $24.6 \pm 1.48$ & $19.7 \pm 0.26$ & $19.2 \pm 0.41$ \\
\hline Nanoemulsion 2\% & $28.2 \pm 1.15$ & $21.0 \pm 0.77$ & $23.7 \pm 0.90$ \\
\hline Blank of cream & $00.0 \pm 0.00$ & $00.0 \pm 0.00$ & $00.0 \pm 0.00$ \\
\hline Cream 1\% & $12.4 \pm 0.80$ & $12.3 \pm 1.20$ & $13.7 \pm 1.38$ \\
\hline Cream 1,5\% & $13.5 \pm 2.98$ & $13.6 \pm 1.52$ & $13.9 \pm 0.15$ \\
\hline Cream 2\% & $17.8 \pm 1.34$ & $17.8 \pm 0.95$ & $18.3 \pm 3.10$ \\
\hline
\end{tabular}


RASĀYAN J. Chem.

Vol. 13 | No. 1 |529 - 534| January - March | 2020

\section{CONCLUSION}

According to the observation, this study resulted in the formula of miconazole nitrate nanoemulsion had a higher cumulative percent penetration and antifungal activity compared to the formula of miconazole nitrate cream. Meanwhile, the pharmacodynamic and pharmacokinetic evaluation of the system in human volunteers is still needed for confirmation.

\section{ACKNOWLEDGMENT}

Authors of this study thanks to Kimia Farma Watudakon Jombang, East Java Indonesia for the miconazole nitrate samples. In addition, we are thankful for the supports from the Faculty of Pharmacy, Universitas Sumatera Utara, Indonesia.

\section{REFERENCES}

1. S. Gungor, M.S. Erdal, B. Aksu, Journal of Cosmetics, Dermatological Sciences and Applications., 3, 56 (2013), DOI: 10.4236/jcdsa.2013.31A009

2. C.M. Lee, H.I. Maibach, Journal of Pharmaceutical Science, 95, 1405 (2006), DOI:10.1002/jps.20666

3. G.S. Sanap, P. Mohanta, International Journal of Pharmacy and Pharmaceutical Sciences., 6, 393 (2014).

4. S. Samira, M.T. Ali, S. Fakhr, D. Saed, M. Hamidrwza, Iranian Journal of Pharmaceticals Sciences, 7, 139 (2015).

5. P.T.P. Kumar, N.K.S. Prakash, P. Lokesh, K. Manral, Journal of Research dan Method in Education, 5, 1 (2015), DOI: 10.9790/7388-05130105

6. P.B. Shinde, Asian Journal of Biomedical and Pharmaceutical Sciences, 3, 33 (2013).

7. K. Gurpreet, S.K. Singh, Indian Journal of Pharmaceutical Sciences, 80, 781 (2018).

8. A. Azeem, M. Rizwan, S. Talegaonkar, M. Aquil, F. Ahmad, Z. Iqbal, AAPS Pharm SciTech, 10, 69 (2009), DOI: 10.1208/s12249-008-9178-x

9. R.S. Vijaya, K. Suresh, S. Kamalakannan, Journal of Chemical and Pharmaceutical Sciences, 1, 92 (2015).

10. Malvern Instruments, Inform White Paper: Dynamic Light Scattering Common Terms Defined, Worcestershire: Malvern Instrument, (2011).

11. H.L. Maha, B.Pharm. Thesis, Department of Pharmaceutical Technology, University of Sumatera Utara, Medan, North Sumatera, Indonesia (2017).

12. A. Sambasivarao, C.S. Rao, H.M. Reddy, World Journal of Pharmaceutical and Medical Research. 2, 99 (2016).

13. M. Balouiri, S. Moulay, K.I. Saad, Journal of Pharmaceutical Analysis, 6, 7 (2016), DOI: 10.1016/j.jpha.2015.11.005

14. C. Rodrigues, K.C. Miranda, F. Pernandes, S. Maria, Revista do Instituto de Medicina Tropical de São Paulo, 51, 9 (2009), DOI: 10.1590/s0036-46652009000100002

15. S. Baboota, S. Faiyaz, A.Alka, S. Alisheikh, Acta Pharmaceutica, 57, 315 (2007), DOI: 10.2478/v10007007-0025-5

16. J. Swarbrick, 2007, Encyclopedia of Pharmaceutical Technology, Third Edition, Volume 1., Informa Healthcare USA, New York, pp. 1561-1564.

17. M.S. Shahid, A. Chowdeswari, Scandinavian Journal of Pharmaceutical Science and Research, 1, 6 (2013).

18. N. Akhtar, M.U. Rehman, H.M.S. Khan, F. Rasool, T. Saeed, G. Murtaza, Tropical Journal of Pharmaceutical Research, 10, 281 (2011), DOI:10.4314/tjpr.v10i3.1

19. B. Iskandar, Karsono, J. Silalahi, International Journal of Pharm Tech Research, 9, 307 (2016).

20. Indonesian Health Ministry, Indonesian Pharmacopoeia. III Edition, Indonesia Director General of Health Care and Medical Devices, Jakarta, Indonesia, p. 546 (1979).

21. Sumaiyah, B. M. Leisyah, Rasayan Journal of Chemistry, 12, 1185(2019), DOI: 10.31788/RJC.2019.1235337

22. H. Panwar, N. Chaudhary, S. Singh, S. Singh, Rasayan Journal of Chemistry, 4, 498 (2011).

23. B. Togatorop, K.R. Sinaga, E. Suwarso, I. Iksen, Rasayan Journal of Chemistry, 11, 516 (2018), DOI: 10.31788/RJC.2018.1122070

[RJC-5553/2019] 